\title{
RECIENTES DESARROLLOS EN LA INVESTIGACIÓN TEXTOLÓGICA*
}

\author{
LUCIANO VITACOLONNA \\ Universidad "Gabriele d'Annunzio" de Chieti-Pescara (Italia) \\ vitaco53@hotmail.com
}

\begin{abstract}
Resumen
In this paper I try to sketch the main textological trends that have developed in the last twenty years. I am especially concerned with the last version of J. S. Petöfi s theory, i.e. the so-called semiotic textology. After shortly dealing with some interdisciplinary questions that regard text research (for instance, the role and the relations of pragmatics and semantics, the importance of proceduralism, the role of several types of logic, the utilization of the concepts of possible world and frame, etc.), I single out the most urgent tasks and aims that semiotic textology has to meet, for example the reformulation of the concept and function of both sign and text / discourse, some critical remarks concerning Chomsky's paradigm, the distinction and explication of different kinds of interpretation (theoretical, structural, procedural, evaluative, etc.), the analyse of multimedial texts and hypertexts, and so on.
\end{abstract}

1. En los últimos veinte años se ha desarrollado considerablemente el trabajo de investigación dedicado al texto y al discurso ${ }^{1}$. Uno de los aspectos más significativos de este desarrollo ${ }^{2}$ es el que corresponde al cambio de orientación llevado a cabo desde la

\footnotetext{
* Agradezco muy cordialmente la revisión formal y estilística de este trabajo al Prof. Dr. Francisco Chico Rico.

1 Esto lo demuestra, entre otras cosas, el gran número de revistas aparecidas en los últimos veinte años (entre paréntesis indico su fecha de nacimiento): Text (1981), Discurso. Teoria y Análisis (1983), Discourse Processes (1986), Discurso (1987), Discourse \& Society (1990), Szemiotikai szövegtan (1990), Texto! (1995), Officina Textologica (1997), Discourse Studies (1999), Critical Discourse Studies (2004), etc. También hay que señalar tanto los numerosos congresos y simposios sobre la investigación textual (vid., por ejemplo, Conte, Petöfi y Sözer (eds.), 1989; Fordyce y Marello (eds.), 1994 y Kronning, 2001: 99, n. 5) como los varios cientos de sitios que conciernen a la lingüística'semiótica del texto, como, por ejemplo, <http:/www.text-semiotics.com> (en febrero de 2001 ya había más de 1.100 sitios). Por último, mucha importancia tienen, igualmente, los "Quaderni di Ricerca e Didattica" de la Universidad de Macerata (Italia) que tratan de los "Sistemi segnici e loro uso nella comunicazione umana" ("Sistemas sígnicos y su empleo en la comunicación humana"), de los cuales hasta ahora se han publicado cinco volúmenes (vid. Petöfi (ed.), 1993; Petöfi y Cicconi (eds.), 1995; Petöfi y Vitacolonna (eds.), 1996; Petöfi y Rossi (eds.), 1997 y Petöfi y Pascucci (eds.), 2001). En cuanto a la definición de los términos texto y discurso, no hay un único punto de vista (vid., a este respecto, Vitacolonna, 1989: 71-98).

2 Una breve historia de la investigación textual se encuentra en Vitacolonna, 1999: 87-107. Vid. también Harweg, 1974; Conte, 1977; Rieser, 1981; Petöfi, 1985, 1990; Beaugrande, 1990, 1997b; Saiz Noeda, 1994; Van Dijk, 1995a; Antos y Tietz (eds.), 1997 y Hölker, 2001.
} 
lingüistica del texto (nacida en Alemania en 1964) a la teoría del texto (es decir, a un enfoque teórico-textual) ${ }^{4}$, lo que ha significado adoptar un punto de vista semiótico (de aquí la semiótica del texto $)^{5}$. Más recientemente, en torno a 1990 , se ha desarrollado un nuevo marco teórico, la llamada textología semiótica, que consituye el último modelo de la teoría de János S. Petöfí.

Aunque no sea oportuno examinar aquí detalladamente la relación entre lingüística del texto y teoria/semiótica del texto ${ }^{7}$, quizá sí es conveniente precisar lo que sigue:

(1) mientras que el término lingüistica del texto destaca el hecho de que el lenguaje existe y puede ser examinado solamente en forma de textos ${ }^{8}$, la teoría del texto pone un especial acento en el objeto de la investigación ${ }^{9}$, esto es, no en el lenguaje-como-texto, sino en el texto-como-complejo-sígnico;

(2) mientras que la lingüistica del texto se interesaba y se interesa sólo por los textos unimediales de naturaleza lingüístico-verbal (admitiendo que existan textos exclusivamente unimediales) ${ }^{10}$, la semiótica del texto se interesa por todas las clases de textos, cualesquiera que sean los media empleados para su configuración (Petöfi y Cicconi (eds.), 1995). En este sentido, se debería ya reconocer y aceptar que, de hecho, no existen textos exclusivamente unimediales (Schröder, 1995: 12) ${ }^{11}$.

3 En efecto, en 1964 Peter Hartmann publicó su célebre artículo "Text, Texte, Klassen von Texten" ( Texto, textos, clases de textos ), donde afirmaba que el lenguaje existe y se puede analizar sólo en forma de textos (Hartmann, 1972). También en 1964 apareció la primera edición del fundamental trabajo de Harald Weinrich sobre los tiempos verbales (Weinrich, 1964). Sobre la lingüística del texto vid. Dressler, 1972; Conte (ed.), 1977; Beaugrande y Dressler, 1981; Bernárdez, 1982; Albaladejo y García Berrio, 1983 y Andorno, 2003.

4 Cfr. Lozano et al., 1993 y La Matina, 2001: 44-51.

5 Por ejemplo, vid. Vitacolonna, 1999 y Pozzato, 2001. Vid. también Talens et al., 1999.

6 Sobre la textología semiótica vid. Petöfi, 1991, 1995a, 1995b, 1996a, 1996c, 2000; La Matina, 1994; Petöfi y Vitacolonna (eds.), 1996; Vitacolonna, 1999; Navarro Colorado y Blanco Fernández, 2000 y Navarro Colorado, 2001. Hay que recordar que las principales versiones de la teoría de János S. Petöfi son: la TeSWeST (= Text-Struktur Welt-Struktur Theorie), la TeSReST (= Text-Struktur Relatum-Struktur Theorie), la VeSReST (= Vehiculum-Struktur Relatum-Struktur Theorie) y, precisamente, la textología semiótica.

7 La relación entre lingüística del texto y teoria/semiótica del texto nos recuerda un poco aquélla entre lingüistica y semiologia/semiótica: ¿es la semiología/semiótica la que incluye a la lingüística o viceversa? De hecho, toda la querelle saussureana-barthesiana tendría que ser reconsiderada partiendo de otras bases, pero esto excede los límites de mi trabajo (para un primer enfoque del problema, vid. Gambarara, 1999: 25-30).

8 Esencialmente ésta era la tesis del "fundador" de la lingüística del texto, Peter Hartmann, que, en 1964, afirmaba: «Mit Text kann man alles bezeichnen, was an Sprache so vorkommt, daß es Sprache in kommunikativer oder wie immer sozialer, d.h. partnerbezogener Form ist; oder kürzer: Sprache kommt beobachtbar vor in Textform; und: Noch einmals ist Sprache in anderer Form als in Textform vorgekommen, d.h. in Sprachfunktion geäußert worden» (Hartmann, 1972: 5). A propósito de Hartmann, vid. Hölker, 2001 y Beaugrande, 1991 (el capitulo sobre Peter Hartmann se puede leer también en la versión electrónica de Linguistic Theory: The Discourse of Fundamental Works: <http://www.beaugrande.com>).

9 Recuerdo, en passant, la distinción entre método y teoría como a menudo ha sido señalada por Prosdocimi (vid., por ejemplo, Prosdocimi, 1979a: 119; 1979b: 1.841, nota 5).

10 Cfr. las primeras investigaciones del llamado "Grupo de Constanza", constituido, entre otros, por J. F. Ihwe, J. S. Petöfi, H. Rieser y M. Rüttenauer (vid. PTK, 1974), o las de Gülich, Heger y Raible (1979). Además, hay que recordar que estas primeras investigaciones a menudo se centraban en el texto literario, considerado como texto por excelencia (vid. Prosdocimi, 1984: 66). Por fin, tenemos que mencionar las varias iniciativas y actividades (investigaciones, congresos, seminarios, etc.) del célebre "Zentrum für interdisziplinäre Forschung (ZiF)" de la Universidad de Bielefeld. 
En efecto, las investigaciones en el ámbito de la lingüística del texto, antes, y de la teoría del texto, después, han probado, como escribe Petöfi, que

un adeguata autolimitazione può essere eseguita soltanto in relazione a una teoria che può al contempo essere organicamente aperta e chiusa (a) da un lato in rapporto alla semiotica generale, alla teoria della comunicazione, alla teoria dell interpretazione e alla fondazione interdisciplinare; (b) dall altro in rapporto a una teoria più ampia e anche alle teorie parziali in essa contenute. A questo riguardo, chiamo la teoria più ampia teoria semiotica della comunicazione umana multimediale, mentre chiamo la teoria adeguatamente autolimitabile teoria della comunicazione umana multimediale ma prevalentemente verbale, in breve testologia semiotica. La prevalenza della verbalità sta a significare che gli elementi prevalentemente costitutivi del significato sono elementi lessicali, con cui, però, possono coagire anche gli elementi costitutivi del significato di qualunque altro medium, come ci testimoniano il contesto della comunicazione quotidiana, la televisione, il film, etc. (Petöfi, 1989-1990: 622).

Pero, como hemos visto, es más correcto reservar -según hace János S. Petöfi (1996a, 1996c) - el término texto sólo para los objetos semióticos con un componente verbal, y utilizar, para los otros casos, el término comunicado ${ }^{12}$;

(3) la textología semiótica es un marco teórico más potente, más amplio y más complejo que la teoría del texto o que la semiótica del texto, y, por supuesto, que la lingüística del texto, ya que la textología semiótica, como afirma Petöfi, «es una disciplina que tiene como objeto la interpretación de los comunicados con (equi)dominio verbal, producidos o recibidos en distintas situaciones comunicativas. Considera los comunicados como complejos sígnicos y la interpretación como análisis y descripción de la arquitectónica formal y de la arquitectónica semántica de los comunicados» (Petöfi, 1996c: 13; 1994: 114) ${ }^{13}$. Por otra parte, «esta disciplina no está vinculada estrechamente ni a un tipo de situación comunicativa ni a un tipo de medium (o de lenguaje natural) ni a un tipo específico de comunicados»» (Petöf, 1996c: 13). Por tanto, aunque venga prefigurada una textología semiótica general, János S. Petöfi (1996c) prefiere hablar de textologías semióticas (en plural).

La antedicha serie de fases de desarrollo -desde la lingüística del texto a la teoría del texto $\mathrm{y}$, finalmente, a la textología semiótica- responde (y lleva) también a un nuevo concepto de texto. Así, un texto puede ser visto:

$(\alpha)$ como un objeto semiótico relacional, es decir, como la manifestación de una relación "significante-significado" (utilizando términos saussureanos), o bien

( $\beta$ ) como un acontecimiento semiósico complejo ${ }^{14}$, esto es, como un acontecimiento formado, al menos, por dos fases fundamentales:

$\left(\beta_{1}\right)$ la producción-construcción del texto como objeto semiótico relacional y $\left(\beta_{2}\right)$ la recepción-interpretación del texto como objeto semiótico relacional.

12 En este punto habría que definir (o redefinir) el concepto de discurso, relegando quizá su empleo al ámbito de la oralidad o de la realización (performance). Pero todo esto excede los límites impuestos a este trabajo.

13 Quiero precisar que la traducción de Petöf (1996c) es la que se encuentra en I. S. Petöf, "El contexto disciplinar de la investigación textológica. Aspectos de la textologia semiótica", en TonosDigital, 1, marzo de 2001: $<$ http://www.um.es/tonosdigital/znuml/estudios/petofi.htm $>$.

14 Sobre el concepto de texto como acontecimiento (event), vid. Beaugrande, 1997a: 10. 
Estas dos fases $-\left(\beta_{1}\right)$ y $\left(\beta_{2}\right)$ - pueden tener lugar o no en el mismo contexto crono-topológico, pero proceden siempre en sentido inverso, pues -según la terminología de Petöfi- la producción parte de un relatum (o de una relatum-imago) y llega a la creación de un vehiculum (la manifestación física de un signo), mientras que la interpretación parte de un vehiculum (o de una vehiculum-imago) y llega a una relatum-imago (o a un relatum).

Aquí es necesario hacer, sin embargo, una precisión. Este modelo comunicativo, formado por las fases $\left(\beta_{1}\right)$ y $\left(\beta_{2}\right)$, puede recordarnos los modelos "clásicos" de la comunicación ${ }^{15}$ $\mathrm{y}$, como aquéllos, puede parecer restrictivo o simplista ${ }^{16}$. Pero, en realidad, el modelo comunicativo elaborado por János S. Petöfi es mucho más complejo y estructurado que aquéllos, ya que no sólo se atiene a la estructura triádica emisor-mensaje-destinatario: toma también en consideración factores como la tipología de las situaciones comunicativas (concretas o supuestas), la intención dominante (que establece la configuración de las funciones comunicativas elegidas/activadas concreta o hipotéticamente en una situación comunicativa determinada), el sistema de los conocimientos (del cual forma parte el conocimiento tipológico que dirige y verifica las bases ), el sistema de las hipótesis y de las disposiciones, la tipología de los comunicados (que pueden ser heteromediales, multimediales o multimediales predominantemente verbales), la tipología de los media (que pueden ser verbales, para-verbales y no-verbales), etc. ${ }^{17}$.

2. Por lo que atañe a los principios o criterios -constitutivos y regulativos- que un texto tiene que satisfacer, no existe un único punto de vista ${ }^{18}$. Sin embargo, podemos afirmar que un productor y/o un intérprete consideran un objeto semiótico (heteromedial, multimedial o multimedial predominantemente verbal) o un acontecimiento semiósico como un texto si creen que ese objeto o ese acontecimiento constituyen una totalidad completa capaz de satisfacer una intención comunicativa concreta o supuesta en una situación comunicativa concreta o supuesta ${ }^{19}$.

Por lo que respecta a la interdisciplinariedad de la investigación textual, ésta tiene que ser vista de manera dialéctica:

(a) por una parte, varias y siempre nuevas disciplinas han contribuido y contribuyen al desarrollo de la investigación textual. Aparte de aquellas que más directamente han dado origen a la lingüística del texto -la retórica, la estilística, el análisis estructural del relato y, parcialmente, la filología $-^{20}$, otras disciplinas -como la semiótica, la ciencia/teoría de la literatura, la psicología (principalmente la psicología cognitiva), la filosofia (principalmente la filosofia analítica), la pragmática, la hermenéutica, la cinésica, la proxémica, etc.- han desempeñado y siguen desempeñando papeles muy importantes;

(b) por otra parte, la investigación textual ha influido e influye notablemente en varios sectores de la investigación científica, como, por ejemplo, en la lingüística, en la sociología,

15 Cfr., por ejemplo, Shannon y Weaver, 1949; Jakobson, 1963 y Volli, 2002.

16 Para una crítica de estos modelos, vid. Finnegan, 2002: 9-17.

17 Vid. Petöfi, 1989-1990, 1995c; Vitacolonna, 1999: 41-44; Navarro Colorado, 2001 y Petöfi y Pascucci, 2001.

18 En cuanto a la distinción entre criterios constitutivos y regulativos, vid. Beaugrande y Dressler, 1981: 11-12; Vitacolonna, 1999: 112 sigs. y Andorno, 2003: 17-21; vid. incluso Beaugrande, 1997a: 13 sigs.

19 Cfr. Petöfi, 1986a: 398 y Navarro Colorado, 2001: 71 sigs.

20 Cfr. Dressler, 1972; Bernárdez, 1982 y Vitacolonna, 1999: 90-92. 
en la etnolingüística, en la antropología cultural, en la inteligencia artificial, en las ciencias cognitivas, en la ciencia de la comunicación, en el estudio de los media, en el análisis de la conversación, en la pedagogía, en la didáctica, etc. (Beaugrande, 1997a). Como escribe Petöfi,

Las textologías semióticas, además de las funciones que tienen en las llamadas macro-disciplinas [...], son también "interdisciplinas" en la medida en que su construcción requiere dar cuenta de los aspectos tratados en la filosofía, la semiótica, la teoría de la comunicación, la psicología, la sociología, etc. (Petöfi, 1996c: 20-21).

Sin embargo, creo que las novedades más relevantes son las que siguen:

(i) la integración de los componentes semántico y pragmático ${ }^{21}$. Esto, además de causar el rechazo de la contraposición chomskiana entre sintaxis y semántica, ofrece a la teoría del texto la única posibilidad concreta de exceder los límites lingüísticos. De hecho, sólo si insertamos el texto en una situación comunicativa dada (en un contexto), podemos explicar fenómenos como el empleo de los deícticos, la (co)referencia no lingüística, la entonación, la gestualidad, la intertextualidad, los turnos dialógicos, el empleo de los registros lato sensu, etc.;

(ii) la tendencia, cada día más frecuente, a reemplazar el estructuralismo y el generativismo por el procedimentalismo. Los análisis procedimentales no rechazan ni desatienden los resultados, las adquisiciones y los méritos de las teorías estructuralistas y generativotransformacionales: el procedimentalismo es solamente un aparato (un marco teórico) más potente para la interpretación de los textos y los discursos. Según señala János S. Petöfi,

In der linguistischen Forschung -in der Erforschung der natürlichsprachlichen Kommunikation im allgemeinen- gilt in verschiedenen Phasen strukturaler Linguistik und generativer Linguistik (um nur diese beiden zu erwähnen) ist mehr und mehr eine linguistische Richtung im Aufkommen, in der der Aspekt Dynamik als der dominierende Aspekt deklariert ist. Diese Linguistik wird zunehmend unter der Bezeichnung prozedurale Linguistik bekannt (Petöfi, 1983: 310).

En otro lugar, además, precisa lo siguiente:

Der Terminus prozedural zeigt bei mir den dynamischen Charakter eines Modells an, unabhängig davon, ob durch dieses Modell der Aufbau eines natürlichen Objektes oder ingerdein auf das betreffende Objekt bezogener Prozeß modelliert werden soll (Petöfi, 1983: 316).

3. A pesar de todos estos resultados en los ámbitos tanto teórico como metodológico, la investigación textológica aún tiene que recorrer un largo camino. Vamos a bosquejar las que -a mi juicio-son sus tareas más urgentes.

(1) La investigación textológica tendría que especificar mejor y de manera más clara la relación que existe, por una parte, entre la semántica y la pragmática (actos de habla incluidos), y, por otra, entre estos dos componentes y la gramática/sintaxis. En otros términos: siempre tendríamos que tomar en consideración el contexto o los contextos de comunicación 
(Petöfi, 1995c), pues los textos siempre son producidos e interpretados en contextos dados, es decir, la producción y la interpretación de los textos/discursos son siempre un fenómeno semántico-pragmático. Así, por una parte, hay que auspiciar una nueva revisión de los actos ilocutivos y perlocutivos, y, por otra, hay que establecer una nueva relación con la retórica ${ }^{22}$ -0 , más exactamente, con las retóricas (Petöfi, 1996c)-, con las ciencias cognitivas, con la sociolingüística, con la etnolingüística, etc.

(2) Con el fin de obtener y/o realizar (crear, construir) interpretaciones no ambiguas, no sólo es útil, sino también indispensable, un metalenguaje, o sea, un lenguaje canónico (Petöfi, 1977, 1982). En los últimos años, muchos investigadores han criticado la utilización de las formalizaciones ${ }^{23}$, y francamente no se puede negar que estas formalizaciones son en gran medida engorrosas y no muy "legibles"; sin embargo, la ciencia (y la investigación textológica es un acontecimiento científico) no puede renunciar a un metalenguaje.

(3) Si hasta los años setenta se privilegiaba en la investigación lingüística la gramática y la sintaxis -tanto que el objetivo principal lo constituía la construcción de gramáticas textuales ${ }^{24}$, en los últimos veinte años se ha destacado cada vez más la relevancia fundamental de la semántica. Entre otras cosas, esto ha implicado:

(a) una definición más precisa de las relaciones entre significado, sentido y forma -así como la descripción y explicación de la manera según la cual construimos el significado (los significados) tanto en el proceso de producción como en el proceso de interpretación-, tomando en consideración la diferencia que hay entre el texto (físico), tal como es producido, y el texto (físico), tal como es recibido/percibido/ interpretado;

(b) la reformulación del concepto de signo (lingüistico): la concepción saussureana de la relación signifiant-signifié ya no es suficiente o satisfactoria ${ }^{25}$, y ya no es adecuada tampoco la noción hjelmsleviana de signo. Si no se puede renunciar al concepto de signo (lingüístico), hay que reformularlo (vid., muy especialmente, el modelo de signo elaborado por Petö $\mathrm{f}^{26}$ ), puesto que ya no se puede considerar el signo como una entidad abstracta, invariable (fija) y aislada ${ }^{27}$ : un signo -0 , más exactamente, un complejo sígnico - es siempre un objeto relacional dinámico co(n)textualizado;

22 Por ello se ha prestado una atención particular a las operaciones retóricas que contribuyen a la creación/organización de los textos, es decir, a la inventio, a la dispositio y a la elocutio. Estas tres partes artis están perfectamente relacionadas entre sí, ya que «componen un sistema de producción de estructura de conjunto referencial y de tex to retórico, dentro del sistema más amplio formado por la totalidad de las operaciones retóricas» (Albaladejo, 1989: 59). En esta dirección proceden los trabajos de A. García Berrio, de T. Albaladejo, de F. Chico Rico, entre otros (vid. Albaladejo, 1988, 1989; Chico Rico, 1989; Albaladejo y Chico Rico, 1998 y Albaladejo, Del Río Sanz y Caballero (eds.), 1998).

23 Vid., por ejemplo, Ponzio, 1998: 27.

24 Por ejemplo, vid. Van Dijk, 1972a; Petöfi, 1971a, 1971b; Van Dijk, Ihwe, Petöfi y Rieser, 1972a, 1972b y Rieser, 1972, 1977.

25 Pero siempre tenemos que preguntarnos de "qué" Saussure estamos hablando. En cuanto al concepto saussureano de signo y de semántica, vid. De Palo, 2001 (y también Prosdocimi, 1984; Avalle, 1995 y Caprettini, 1997).

26 Por ejemplo, vid. Petöfi, 1996b.

27 Sin embargo, el mismo Saussure reconoce que «nous ne parlons pas par signes isolés, mais par groupes de signes, par masses organisées qui sont elles-mêmes des signes» (Saussure, 1965: 177). 
(c) la integración de varios tipos de lógica (lógica modal, lógica de los modelos, etc.);

(d) el empleo de conceptos como los de mundo posible, modelo mental, script y frame;

(e) la conexión entre las neurociencias (principalmente las cognitivas) y la investigación en el campo de la inteligencia artificial;

(f) una manera nueva de examinar tanto el problema de la interpretación simbólica, metafórica y figurada ${ }^{28}$ como el problema de la connotación de las extensiones y de las intensiones (Vitacolonna, 1991);

(g) last but not least, una crítica cada vez más severa del marco teórico chomskiano, en el que, sin embargo, se inspiraban o basaban los primeros modelos de gramáticas textuales ${ }^{29}$. Me extenderé algo en este último punto. La razón por la cual la investigación textológica se ha alejado de la lingüística generativo-transformacional se explica si tenemos en cuenta lo que sigue ${ }^{30}$ :

(i) mientras que la lingüística generativo-transformacional ${ }^{3 !}$, en sus varios desarrollos (teoría estándar extendida, semántica generativa, teoría de los principios y parámetros, Gramática Universal, etc.), prescinde siempre de la performance (realización), la lingüística procedimental y la textología semiótica hacen referencia siempre a un emisor y/o a un destinatario -real o supuesto-, esto es, inserto/considerado en un contexto comunicativo concreto o (re)construido;

(ii) la gramática chomskiana se basa en el llamado structure-dependence principle; en cambio, para explicar el proceso comunicativo y el interpretativo, la textología semiótica y la lingüística procedimental siempre toman en consideración la semántica, la pragmática y el ámbito funcional (o bien los ámbitos funcionales);

(iii) mientras que (el último) Noam Chomsky afirma que, aunque el lenguaje pueda ser utilizado para comunicar, "communication is not the function of language», ya que «human languages are biological objects» (Chomsky, 2002: 126) ${ }^{32}$,

28 Con respecto a este tema, vid. Petöfi, 1984a, 1984b, $1986 \mathrm{~b}$.

29 Me refiero, especialmente, al Arbeitsstelle Strukturelle Grammatik, del antiguo Berlín oriental, así como a algunos de los primeros trabajos de Petöfi (1971a, 1971b) y de Van Dijk (1972b). Cfr. también Rieser, 1972.

30 Aquí no quiero ni puedo discutir (a) si es la sintaxis la que determina la estructuración semántica de oraciones, textos, discursos, etc., (b) si es la semántica la que determina la estructuración sintáctica, (c) si hay autonomía entre sintaxis y semántica (como cree Chomsky), o (d) si «the construction of syntax and semantics must procede hand in hand» (Montague, 1974: 210). Sobre la revisión crítica de la autonomía entre sintaxis y semántica en el marco chomskiano, vid. Delfitto, 2002.

31 Salkie llama la atención sobre «the question of what a better label might be for Chomsky s linguistics. Chomsky often describes his enterprise as using language to investigate cognitive aspects of human nature, but this is a long mouthful and we may be left with no alternative to the expression Chomsky s linguistics $\gg$ (Salkie, 2001: 278).

32 Al contrario, las más recientes investigaciones (psicológicas, neurológicas y paleontológicas) sobre el cerebro indican que no hay un órgano biológico para el lenguaje (cfr. Arsuaga, 1999: cap. 9); por otra parte, el mismo Moro (2002) se ve obligado a reconocer que no hay ningún gen de la gramática (cfr. Boncinelli, 2003). Sin embargo, aquí no puedo -ni me interesa tampoco- examinar en detalle los conceptos de Chomsky; una desmitificación de la manera chomskiana de argumentar -desde el punto de vista tanto lingǘstico como político- se encuentra en Beaugrande, 1998 (cfr. también las críticas de Rosiello, 1967; Timpanaro, 1975: 197-209; Coseriu, 1975; Hagège, 1976; Koerner, 1984; Belardi, 1990: 93-153; 1994 y Auroux, 1996). También hay que subrayar que incluso un investigador "proChomsky" como Pinker considera el lenguaje como una adaptación biológica para comunicar informaciones (vid. Pinker, 1994: cap. 1). Semejante al punto de vista de Chomsky es el marco teórico del constructivismo radical (vid. Maturana, 1982; Schmidt, 1990 y Chico Rico (ed.), 1995; vid. también Potebnja, 1895). 
$\mathrm{y}$, según destaca Moro, «i meccanismi biologici che sottendono al linguaggio sono neutrali rispetto alla comunicazione» (Moro, 1995: 670) ${ }^{33}$, para la textología semiótica y la lingüística procedimental el objetivo general y fundamental es precisamente el estudio y análisis de la comunicación humana en todas sus tipologías o formas y de todos los sistemas sígnicos (el lenguaje in primis) utilizados por los seres humanos, incluso porque -como ya hemos dicho- los textos son siempre comunicados multimediales.

(4) Hasta hace unos años, la investigación textológica hacía frente sólo a breves (fragmentos de) textos o discursos ${ }^{34}$. Ahora se propone (conseguir) examinar textos, discursos, hipertextos e hipermedia ${ }^{35}$ concretos (reales) de diferente tamaño y complejidad. Ello implica:

(a) elaborar una estrategia potente y sofisticada capaz de simular cómo funcionan nuestras mentes (nuestros cerebros) en el proceso interpretativo; con respecto a esto, es muy importante el proceso de la reinterpretación (local y global) ${ }^{36}, \mathrm{y}$, sobre todo, la reinterpretación de textos/discursos orales. Las preguntas fundamentales son: dónde/cuándo/ por qué empeza la reinterpretación, y cómo procede;

(b) considerar tanto la relación entre tema y rema (topic-comment) como las presuposiciones y las inferencias ${ }^{37}$ como mecanismos aptos para asegurar la coherencia textual y la funcionalidad pragmática;

(c) examinar (fragmentos de) textos y discursos concretos (reales), producidos oralmente o por escrito en situaciones comunicativas concretas (conversaciones, diálogos, debates, conferencias, actividades didácticas, coloquios entre médico y paciente, discusiones familiares o escolásticas, artículos periodísticos, ensayos científicos, anuncios publicitarios, cuñas, mensajes propagandísticos, recetas, relatos, novelas, cuentos, chistes, graffiti, mensajes enviados electrónicamente por medio de ordenadores o móviles, etc.). En este ámbito entra también el Critical Discourse Analysis ( análisis critico del discurso) ${ }^{38}$, que, al considerar los discursos (y los textos) como un tipo de práctica social (social practice), estudia ejemplos concretos de interacción social que asumen un aspecto -al menos en parte- lingüístico (Wodak, 1997: 173). Fairclough y Wodak escriben a este respecto lo siguiente:

Discursive practices may have major ideological effects: that is, they can produce and reproduce unequal power relations between (for instance) social classes, women and men, and ethnic/cultural majorities and minorities through the ways in which they represent things and position people (Fairclough y Wodak, 1997: 258);

33 Es claro que el lenguaje puede ser utilizado también con otras intenciones (por ejemplo, para describir y reproducir la realidad, para organizar los conocimientos, para dirigir las maneras de comportarse, para dar órdenes, etc.), que son diferentes de la comunicación lingüística stricto sensu (vid. Cardona, 1976, 1985a, 1985b, 1990; Duranti, 1997 y Turchetta (ed.), 1996).

34 Por ejemplo, vid. Eikemeyer y Rieser, 1985 y Vitacolonna, 1999: 127-155.

35 Sobre los hipertextos, vid. Petöfi y Rossi (eds.), 1997 y Landow, 1998.

36 Cfr. Vitacolonna, 1999: 105-106.

37 Cfr. van de Velde, 1992; Bertuccelli Papi, 2000 y Andorno, 2003.

38 Cfr. De Cillia, Reisigl y Wodak, 1999; Fairclough, 1985, 1989; Fairclough y Wodak, 1997; Van Dijk, 1990, 1994, 1995b; Van Dijk (ed.), 1997a, 1997 y y Wodak, 1995a, 1995b, 1997. 
(d) considerar los textos y los hipertextos como objetos dinámicos multimediales ${ }^{39}$, que tienen o pueden tener una organización no lineal, y que son construidos (o se construyen) durante el proceso interpretativo (Rossi (ed.), 2000).

(5) Por lo que respecta a la interpretación, con referencia a János S. Petöfi (2001: 27-28), podemos especificar lo que sigue:

(a) ante todo, hay que distinguir entre interpretación natural e interpretación teórica; mientras que la primera es realizada de manera intuitiva por un intérprete medio en una situación comunicativa normal, la segunda es realizada partiendo de un marco teórico;

(b) la interpretación teórica, a su vez, puede ser explicativa o evaluativa; el objetivo de la interpretación explicativa es la atribución al vehiculum de una arquitectónica formal y semántica, mientras que el objetivo de la interpretación evaluativa es la evaluación de estas arquitectónicas con relación a un sistema de normas determinado (es decir, desde un punto de vista histórico, ideológico, estético, moral, etc.);

(c) la interpretación explicativa, a su vez, puede ser estructural, por representar solamente las relaciones estáticas entre los elementos de las arquitectónicas, o bien procedimental, por representar las relaciones entre los elementos de las arquitectónicas y además las informaciones atinentes a la construcción lógico-temporal de dichas relaciones;

(d) además, la interpretación explicativa -como la evaluativa- puede ser descriptiva, si representa los resultados sin suministrar de manera explícita los fundamentos teóricos que han llevado a dicha interpretación, o bien argumentativa, si ofrece de manera explícita los argumentos a favor de dicha interpretación;

(e) por último, la interpretación explicativa -que puede ser profesional o personalpuede ser de primer grado o de segundo grado, y la de primer grado puede ser también parcialmente figurada. Como precisa Petöfi,

Parliamo di interpretazione di primo grado quando ogni elemento costitutivo di un dato vehiculum può essere interpretato con il suo significato "letterale", come nel caso in cui si ha a che fare con i testi delle cosiddette scienze formali. Parliamo di un interpretazione parzialmente figurativa quando alcuni elementi costitutivi di un dato vehiculum debbono essere interpretati in modo figurativo, mentre ogni altro elemento può essere interpretato col suo significato letterale, come accade nella maggior parte dei testi. E infine, parliamo di interpretazione di secondo grado quando assegniamo a un dato vehiculum un tipo qualsiasi di significato simbolico, indipendentemente dal fatto che questo vehiculum possa anche ricevere un interpretazione di primo grado o una parzialmente figurativa; ciò accade quando abbiamo a che fare con testi poetici e/o mitici (Petöfi, 2001: 28).

(6) La investigación textológica y la textología semiótica tienen que distinguir y describir los diferentes tipos de textos. Sin embargo, puesto que lo que llamamos "tipología" no es una cualidad o propiedad intrínseca de los textos, sino una función atribuida a los mismos de acuerdo con criterios que varían en el tiempo y en el espacio, las tipologías y las taxonomías textuales tendrían que ser consideradas como cuestiones tanto pragmático-semánticas como 
pragmático-formales. Entre los diferentes tipos de textos, los literarios merecen una atención muy especial por varios motivos: su ontologia, sus funciones, su empleo didáctico, etc.

(7) La textología semiótica tiene que dar cuenta de los nuevos sistemas de comunicación, ventajas y desventajas incluidas. Con respecto a esto, Riva se expresa en los siguientes términos:

All inizio del terzo millennio i processi di comunicazione appaiono profondamente caratterizzati dall influenza delle nuove tecnologie di comunicazione che hanno condotto alla realizzazione di un sistema di artefatti denominati collettivamente come new media. In particolare, insieme alla telefonia cellulare, la grande diffusione del personal computer e di Internet sta modificando il modo di comunicare della specie umana. Da una parte, 1 uso della Rete (o Internet) ha introdotto nuovi modelli di comunicazione attraverso modalità d interazione assai diverse rispetto alla comunicazione faccia-a-faccia. Nel medesimo tempo ha trasformato il rapporto esistente tra soggetto e tecnologie informatiche: grazie a Internet il personal computer ha gradualmente perso la propria centralità trasformandosi sempre di più da archivio o da calcolatore a uno strumento do comunicazione (Riva, 2002: 363).

Como es bien sabido, en la base de estas nuevas tecnologías de la comunicación se encuentra la codificación digitalizada de la información ${ }^{40}$. La digitalización de la información -según Riva (2002: 364) - tiene muchas ventajas: (i) la información digital se puede modificar y memorizar más fácilmente; (ii) la transmisión de las informaciones no está muy sujeta a perturbaciones; (iii) canales diferentes se pueden integrar con mayor facilidad, etc. Sin embargo, como releva Simone (2001: 46), los textos digitales presentan unas características no precisamente positivas: (a) la inmaterialidad («ll testo digitale è immateriale. Non ha bisogno di depositarsi su un supporto di carta o di altra sostanza stabile, non forma volume né massa, non si tocca e non si accumula»); (b) la acontextualidad (el texto digital «non conserva alcuna indicazione circa il sito in cui è stato prodotto»); (c) la falta de personalidad (el texto digital «non porta tracce fisiche della persona che lo ha scritto»).

Gracias, pues, al éxito de los nuevos sistemas de comunicación electrónica y de la comunicación a través del ordenador (Internet, correo electrónico, fax, hipertextos multimediales o hipermedia, sistemas para enviar breves mensajes e imágenes electrónicos por medio de ordenadores o móviles, realidad virtual, maleabilidad de las estructuras arquitectónicas, situaciones interactivas a distancia, etc. $)^{41}$, el concepto mismo de texto (o discurso) podría, si no ser abandonado, sí ser modificado en un sentido más elástico y adecuado a las nuevas situaciones comunicativas e interpretativas, cada día más complejas y dinámicas. Como escribe János S. Petöfi,

En la comunicación cotidiana, como en la científica, tenemos que producir y recibir/interpretar discursos/textos o, utilizando una expresión más adecuada, "comunicados multimediales" orales o escritos/impresos, que no siempre contienen un componente verbal en una sola lengua natural. Dado que el uso de los media (y de su combinación) ha sufrido en los últimos años una evolución tan repentina como rápida, dichos procesos comunicativos exigen hoy una competencia comunicativa casi autónoma, es decir, no vinculada estrechamente ni a un sector específico de la comunicación, ni a un medium y/o lengua

41 Con respecto a esto, vid. también Rossi (ed.), 2000; Paccagnella, 2000 y Simone, 2001. 
natural específica. La necesidad de esta competencia se hace más evidente si consideramos el hecho de que cada vez más personas usan la "video-escritura multimedial" en sus ordenadores personales (Petöfi, 1996c: 20).

De acuerdo con todo lo que hemos dicho, podemos distinguir diferentes objetivos fundamentales en la investigación textológica. Sin embargo, creo que la tarea más urgente de la investigación textológica es la de explicar cómo funciona el proceso interpretativo. En efecto, la diferencia verdadera entre los seres humanos, por una parte, y los animales y los ordenadores, por otra, es que los animales y los ordenadores pueden (quizá) interpretar mensajes o inputs, mientras que los seres humanos pueden y tienen que explicar la interpretación misma, no solamente como resultado, sino especialmente como proceso.

\section{Referencias bibliográficas}

Albaladejo, T. (1988): "Semántica y sintaxis del texto retórico: inventio, dispositio y partes orationis", en Estudios de Lingüistica, 5, págs. 9-15.

Albaladejo, T. (1989): Retórica, Madrid, Síntesis.

Albaladejo, T. y Chico Rico, F. (1998): "La Intellectio en la serie de las Operaciones Retóricas no Constituyentes de Discurso", en Albaladejo, Del Río Sanz y Caballero (eds.) (1998), págs. 339352.

Albaladejo, T., Del Río Sanz, E. y Caballero, J. A. (eds.) (1998): Retórica hoy (= Teoria/Crítica, 5), Alicante, Universidad de Alicante.

Albaladejo, T. y García Berrio, A. (1983): "La lingüística del texto", en Abad, F. y García Berrio, A (eds.): Introducción a la lingüistica, Madrid, Alhambra, 1983, págs. 217-260.

Andorno, C. (2003): Linguistica testuale. Un'introduzione, Roma, Carocci.

Antos, G. y Tietz, H. (eds.) (1997): Die Zukunft der Textlinguistik, Tübingen, Niemeyer.

Arsuaga, J. L. (1999): El collar del Neandertal, Madrid, Ediciones Temas de Hoy.

Auroux, S. (1996): La philosophie du langage, Paris, PUF.

Avalle, D A. S. (1995): Ferdinand de Saussure fra strutturalismo e semiologia, Bologna, Il Mulino.

Beaugrande, R. de (1990): "Text linguistics through the years", en Text, 10/1-2: 9-17.

Beaugrande, R. de (1991): Linguistic Theory: The Discourse of Fundamental Works, London, Longman.

Beaugrande, R. de (1997a): New Foundations for a Science of Text and Discourse, Norwood (N. J.), Ablex.

Beaugrande, R. de (1997b): “Textlinguistik: Zu Neuen Ufern?”, en Antos y Tietz (eds.) (1997), págs. $1-12$.

Beaugrande, R. de (1998): "Performative speech acts in linguistic theory: The rationality of Noam Chomsky", en Journal of Pragmatics, 29, págs. 1-39.

Beaugrande, R. de (en prensa): "There s No Such Thing As Syntax - And It s a Good Thing, Too ...", en Hladky, J. et al. (eds.): Festschrift in Honour of Jan Firbas, Amsterdam, Benjamins (vid. http://beaugrande.bizland.com/FirbasFest.htm).

Beaugrande, R. de y Dressler, W. U. (1981): Introduction to Text Linguistics, London/New York, Longman.

Belardi, W. (1990): Linguistica generale, flologia e critica dell'espressione, Roma, Bonacci.

Belardi, W. (1994): "Il ruolo del segno linguistico nel sapere nozionale e nel pensare discorsivo", en Negri, M. y Poli, D. (eds.): La semantica in prospettiva diacronica e sincronica, Pisa, Giardini Editori, 1994, págs. 75-96.

Bernárdez, E. (1982): Introducción a la lingüística del texto, Madrid, Espasa Calpe. 
Bertuccelli Papi, M. (2000): Implicitness in Text and Discorse, Pisa, Edizioni ETS.

Boncinelli, E. (2003): "I presupposti biologici del linguaggio, I. Aspetti evolutivi", in Lingue e Linguaggio, II, 1, págs. 147-159.

Caprettini, G. P. (1997): Segni, testi, comunicazione, Torino, UTET Libreria.

Cardona, G. R. (1976): Introduzione all'etnolinguistica, Bologna, Il Mulino.

Cardona, G. R. (1985a): La foresta di piume. Manuale di etnoscienza, Roma/Bari, Laterza.

Cardona, G. R. (1985b): I sei lati del mondo. Linguaggio ed esperienza, Roma/Bari, Laterza.

Cardona, G. R. (1990): I linguaggi del sapere, Roma/Bari, Laterza.

Chico Rico, F. (1989): “La intellectio. Notas sobre una sexta operación retórica”, en Castilla. Estudios de literatura, 14 , págs. 47-55.

Chico Rico, F. (ed.) (1995): La Ciencia Empírica de la Literatura. Conceptos, métodos, consecuencias (= Teoria/Crítica, 2): Alicante, Universidad de Alicante.

Chomsky, N. (2002): On Nature and Language, Cambridge, CUP.

Conte, M.-E. (1977): "Introduzione" a Conte (ed.) (1977): 11-50.

Conte, M.-E. (ed.) (1977): La linguistica testuale, Milano, Feltrinelli.

Conte, M.-E., Petöfi, J. S. y Sözer, E. (eds.) (1989): Text and Discourse Connectedness. (Proceedings of the Conference on Connexity and Coherence - Urbino, July 16-21, 1984), Amsterdam/Philadelplhia, Benjamins.

Coseriu, E. (1975): Leistung und Grenzen der transformationellen Grammatik, Tübingen, Narr.

De Cillia, R., Reisig1, M. y Wodak, R. (1999): “The discursive construction of national identities”, en Discourse and Society, 10 (2): 149-173.

Delfitto, D. (2002): "Linguistica chomskiana e significato. Valutazioni e prospettive", en Lingue $e$ Linguaggio, I, 2: 197-236.

De Palo, M. (2001): La conquista del senso. La semantica tra Bréal e Saussure, Roma, Carocci.

Dressler, W. U. (1972): Einführung in die Textlinguistik, Tübingen, Niemeyer.

Duranti, A. (1997): Linguistic anthropology, Cambridge, CUP.

Eikmeyer, H.-J. y Rieser, H. (1985): "Procedural grammar for a fragment of Black English Discourse", en Ballmer, Th. T. (ed.): Linguistic Dynamics. Discourses, Procedures and Evolution, Berlin/New York, W. de Gruyter, 1985, págs. 85-178.

Fairclough, N. (1985): "Critical and descriptive goals in discourse analysis", en Journal of Pragmatics, 9, págs. 739-763.

Fairclough, N. (1989): Language and Power. London, Longman.

Fairclough, N. y Wodak, R. (1997): "Critical Discourse Analysis", en Van Dijk (ed.) (1997b): 258-284.

Finnegan, R. (2002): Communicating. The Multiple Modes of Human Interconnection, London/New

York, Routledge.

Fordyce, R. y Marello, C. (eds.) (1994): Semiotics and Linguistics in Alice's Worlds. Proceedings of the

Conference on "La linguistica di Alice" - Urbino, July 10-12, 1990, Berlin/New York, W. de Gruyter.

Galli, G. (ed.) (1984): Interpretazione e simbolo, Genova, Marietti.

Gambarara, D. (1999): "Significato e segno. Per una semantica del significato", en Gambarara, D. (ed.): Semantica, Roma, Carocci, 1999: 25-45.

Gülich, E., Heger, K. y Raible, W. (1979): Linguistische Textanalyse. Überlegungen zur Gliederung von Texten, Hamburg, Buske.

Hagège, C. (1976): La grammaire génèrative. Réflexiones critiques, Paris, PUF:

Hartmann, P. (1972): "Text, Texte, Klassen von Texten", en Koch, W. (ed.): Strukturelle Textanalyse - Analyse du récit - Discourse Analysis, Hildesheim/New York, Olms, 1972: 1-22 (edición orig. En Bogawus, 2, 1964, págs. 15-25).

Harweg, R. (1974): "Textlinguistik", en Koch, W. A. (ed.): Perspektiven der Linguistik II, Stuttgart, Kröner, 1974, págs. 88-116.

Heydrich, W. y Petöf, J. S. (1993): "Towards a General Pragmatics of Language", en Stachowiak, H. (ed.): Pragmatik. Handbuch pragmatischen Denkens, Hamburg, Felix Meiner, 1993, págs. 123155. 
Hölker, K. (2001): “Per costruire un contesto: gli inizi della Textlinguistik”, en Prandi y Ramat (eds.) (2001), págs. 63-79.

Jakobson, R. (1960): “Lingüística y Poética”, en Jakobson, R., Ensayos de lingüística general, Barcelona, Seix Barral, 1975, págs. 347-395.

Koerner, K. (1984): "The Chomskyan Revolution and its historiography", en Arcaini, E, Cigada, S. y Rigotti, E. (eds.): Diacronia, sincronia e cultura. Saggi linguistici in onore di Luigi Heilmann, Brescia, La Scuola, 1984, págs. 153-177.

Kronning, H. (2001): “Au-delà du déontique et de 1 épistémique”, en Prandi y Ramat (eds.) (2001), págs. $97-118$.

La Matina, M. (1994): Il testo antico, Palermo, L epos.

La Matina, M. (2001): Il problema del significante, Roma, Carocci.

Landow, G. P. (1997): Hypertext 2.0. The Convergence of Contemporary Critical Theory and Technol$o g y, 2^{\text {nd }}$ edition, Baltimore, The Johns Hopkins University Press.

Lozano, J. et al. (1993): Análisis del discurso. Hacia una semiótica de la interacción textual, $4^{\text {a edi- }}$ ción, Madrid, Cátedra.

Maturana, H. R. (1982): Erkennen: Die Organisation und Verkörperung von Wirklichkeit. Ausgewählte Arbeiten zur biologischen Epistemologie, Braunschweig/Wiesbaden, Vieweg.

Montague, R. (1970): “English as a Formal Language”, en Montague, R., Formal Philosophy. Selected Papers of Richard Montague, Edited by R. H. Thomason, New Haven/London, Yale University Press, 1974, págs. 188-221.

Moro, A. (1995): "«Virtual conceptual necessity»: la semplificazione della grammatica generativa nei primi anni novanta", en Lingua e Stile, 30/4, págs. 637-674.

Moro, A. (2002): "Linguistica mendeliana ovvero quali domande su genetica e grammatica?", en Lingue e Linguaggio, I, 1: 39-58.

Navarro Colorado, B. (2001): Introducción a la Textología Semiótica. Bases teóricas para la consideración multimedial del texto, Alicante, Universidad de Alicante.

Navarro Colorado, B. y Blanco Fernández, M. S. (2000): "Retórica y Multimedialidad: Aproximación a las relaciones entre la retórica clásica y la textología semiótica", en Revista de Humanidades, I, 1 .

Paccagnella, L. (2000): La comunicazione al computer, Bologna, Il Mulino.

Petöfi, J. S. (1971a): "Transformationsgrammatiken und die grammatische Beschreibung von Texten", en Linguistische Berichte, 14, págs. 17-33.

Petöfi, J. S. (1971b): Transformationsgrammatiken und eine ko-textuelle Texttheorie. Grundfragen und Konzeptionen, Frankfurt am Main, Atenäum.

Petöfi, J. S. (1977): "Lexikoneintragungen in der kanonischen Repräsentation von Sätzen und Texten", en Petöfi, J. S. y Bredemeier, J. (eds.): Das Lexikon in der Grammatik, die Grammatik im Lexikon, Hamburg, Buske, 1977, págs. 297-314.

Petöfi, J. S. (1982): "Representation languages and their function in text interpretation", en Allén, S. (ed.): Text Processing. Text Analysis and Generation, Text Typology and Attribution. Proceedings of Nobel Symposium 51, Stockholm, Almqvist e Wiksell International, 1982, págs. 85-122.

Petöfi, J. S. (1983): "Aufbau und Prozeß, Struktur und Prozedur: Einige Grundfragen der prozeduralen Modelle des Sprachsystems und der natürlich-sprachlichen Kommunikation", en Petöfi, J. S. (ed.): Texte und Sachverhalte: Aspekte der Wort- und Textbedeutung, Hamburg, Buske, 1983, págs. $310-321$.

Petöfi, J. S. (1984a): "Le metafore nella comunicazione quotidiana e nei testi scientifici, biblici e letterari”, en Galli (ed.) (1984), págs. 97-132.

Petöfi, J. S. (1984b): "Interpretazione di un testo e attribuzione di significati simbolici. Annotazioni al Frammento XXXVII di Leopardi”, en Galli (ed.) (1984), págs. 133-154.

Petöfi, J. S. (1985): "La ricerca sulla testologia semiotica in Europa. Una guida storica, tematica e bibliografica", en Studi Italiani di Linguistica Teorica e Applicata, XIV (1/3), págs. 371-400. 
Petöfi, J. S. (1986a): "I parallelismi di Jakobson dalla prospettiva di una teoria testuale semiotica", en Lingua e Stile, XXI, 2-3, págs. 397-426.

Petöfi, J. S. (1986b): "Interpretazione letterale e figurata, intertestualità. Per 1 interpretazione della parabola de Il figliol prodigo ", en Galli, G. (ed.): Interpretazione e invenzione, Genova, Marietti, 1986: $125-142$.

Petöfi, J. S. (1989-1990): "Verso una teoria e filosofia semiotica della comunicazione umana prevalentemente verbale", en Annali della Facoltà di Lettere e Filosofia, Università di Macerata, XXIIXXIII, págs. 621-641.

Petöf, J. S. (1990): "From the analysis of literary works towards a semiotic theory of multimedia human communication. 25 years of textological rsearch: In retrospect and future outlook", en Text, 10/1-2, págs. 73-80.

Petöfi, J. S. (1991): “Aspects of Text Signification. A Semiotic-Textological Approach”, en Petöfi, J. S., Towards a Semiotic Theory of the Human Communication (Text Linguistics - Semiotic Textology), Szeged, Gold Press, 1991: 110-148.

Petöf, J. S. (1994): "Testologia semiotica e filosofia", en Costa, F. y Marrone, G. (eds.): Il testo filosofico, Palermo, L epos, 1994, págs. 113-132.

Petöfi, J. S. (1995a): "La textologie sémiotique et la méthodologie de la recherche linguistique", en Cahiers de l'ILSL, 6, págs. 213-236.

Petöfi, J. S. (1995b): "Die semiotische Testologie und die pragmatichen Aspekte der Kommunikation", en Kertész, A. (ed.): Metalinguistica. Sprache als Kognition - Sprache als Interaktion. Studien zum Grammatik-Pragmatik-Verhältnis, Frankfurt am Main/Berlin/Bern/New York/Paris/Wien, Peter Lang, 1995: 59-100.

Petöfi, J. S. (1995c): "Sulla tipologia delle situazioni comunicative", en Petöfi y Cicconi (eds.) (1995), págs. 25-34.

Petöfi, J. S. (1996a): "La lingua come mezzo di comunicazione scritta: il testo", en Petöfi y Vitacolonna (eds.) (1996), págs. 66-107.

Petöfi, J. S. (1996b): "Dal testo alla comunicazione multimediale - Dalla linguistica alla testologia semiotica della multimedialità", en Petöfi y Vitacolonna (eds.) (1996): 51-65.

Petöfi, J. S. (1996c): "Retorica - Testologia semiotica - Studium generale", en Annali della Facoltà di Lettere e Filosofia, Università di Macerata, XXIX: 9-22.

Petöfi, J. S. (2000): "Teorie del testo e analisi di testi filosofici", en Petöfi, J. S. y Proietti, O., Leggere testi filosofici 1. Aspetti dell'interpretazione (= "Quaderni di Ricerca e Didattica", XIX): Macerata, Università di Macerata, 2000: 64-93.

Petöfi, J. S. (2001): "Alcuni aspetti della Testologia Semiotica. Modello segnico - Tipi di interpretazione”, en Petöfi y Pascucci (eds.) (2001), págs. 21-30.

Petöfi, J. S. (ed.) (1993): Sistemi segnici e loro uso nella comunicazione umana. 1. Aspetti generali. Quadro interdisciplinare della ricerca (= "Quaderni di Ricerca e Didattica", IX): Macerata, Università di Macerata.

Petöfi, J. S. y Pascucci, G. (2001): “Tipologia dei comunicati costituiti da un componente verbale ed uno pittoriale", en Petöfi y Pascucci (eds.) (2001), págs. 31-41.

Petöfi, J. S. y Cicconi, S. (eds.) (1995): Sistemi segnici e loro uso nella comunicazione umana. 2. La filosofia del linguaggio e la comunicazione umana multimediale (= "Quaderni di Ricerca e Didattica", XIV): Macerata, Università di Macerata.

Petöfi, J. S. y Pascucci, G. (eds.) (2001): Sistemi segnici e loro uso nella comunicazione umana.5. Comunicazione visiva: parole e immagini in comunicati statici (= "Quaderni di Ricerca e Didattica", XX): Macerata, Università di Macerata.

Petöf, J. S. y Rieser, H. (eds.) (1973): Studies in Text Grammar, Dordrecht/Boston, Reidel.

Petöfi, J. S. y Rossi, P. G. (eds.) (1997): Sistemi Segnici e loro uso nella comunicazione umana. 4. Combinatoria ed ipertestualità nella ricerca e nella didattica (= "Quaderni di Ricerca e Didattica", XVIII): Macerata, Università di Macerata. 
Petöfi, J. S. y Vitacolonna, L. (eds.) (1996): Sistemi Segnici e loro uso nella comunicazione umana

3. La Testologia Semiotica e la comunicazione umana multimediale (= "Quaderni di Ricerca e Didattica", XVII): Macerata, Università di Macerata.

Pinker, S. (1994): The language instinct, New York, Harper Collins.

Ponzio, A. (1998): "Introduzione" a Rossi-Landi, F., Significato, comunicazione e parlare quotidiano, Venezia, Marsilio, 1998.

Potebnja, A. A. (1895): "Lezione VIII: la parola non comunica il pensiero. Il significato della parola per il parlante stesso. In che cosa consiste la comprensione dell ascoltatore", en Avalle, D A. S. (ed.): La cultura nella tradizione russa del XIX e XX secolo, Torino, Einaudi, 1982, págs. 135141.

Pozzato, M. P. (2001): Semiotica del testo. Metodi, autori, esempi, Roma, Carocci.

Prandi, M. y Ramat, P. (eds.) (2001): Semiotica e linguistica. Per ricordare Maria Elisabeth Conte, Milano, Franco Angeli.

Prosdocimi, A. L. (1979a): "Le iscrizioni italiche: acquisizioni tempi problemi", en Le iscrizioni prelatine in Italia, Roma, Accademia Nazionale dei Lincei, 1979, págs. 119-204.

Prosdocimi, A. L. (1979b): "Lingua e preistoria. Appunti di lavoro", en $\phi \iota \lambda \iota \alpha \varsigma \chi \alpha \rho \iota v$. Miscellanea in onore di Eugenio Manni, Roma, Bretschneider, 1979.

Prosdocimi, A. L. (1984): "Su testo e segno", en Coveri, L. (ed.): Linguistica testuale, Roma, Bulzoni, 1984, págs. 63-84.

PTK (Projektgruppe Textlinguistik Konstanz) (1974): Probleme und Perspektiven der neueren textgrammatischen Furschung I, Hamburg, Buske.

Rieser, H. (1972): "Probleme der Textgrammatik II: Zum Aufbau einer Textgrammatik (TEG)", en Folia Linguistica, 6, págs. 28-46.

Rieser, H. (1977): Textgrammatik, Schulbuchanalyse, Lexikon, Hamburg, Buske.

Rieser, H. (1981): "On the development of text grammar", en Dorfmüller-Karpusa, K. y Petöfi, J. S. (eds.) (1981): Text, Kontext, Interpretation. Einige Aspekte der texttheoretischen Forschung, Hamburg, Buske, 1981, págs. 317-354.

Riva, G. (2002): "Comunicazione e new media", en Anolli, L. (ed.): Psicologia della comunicazione, Bologna, Il Mulino, 2002: 363-383.

Rosiello, L. (1967): Linguistica illuminista, Bologna, Il Mulino.

Rossi, P. G. (ed.) (2000): Dal testo alla rete. Multimedialità e didattica, Napoli, Tecnodid.

Saiz Noeda, M. B. (1994): De la gramática transfrástica a la lingüística comunicativa: nacimiento y desarrollo de la lingüistica del texto, Alicante, Universidad de Alicante.

Salkie, R. (2001): "Transformational Grammar", en Cobley, P. (ed.): The Routledge Companion to Semiotics and Linguistics, London/New York, Routledge, 2001. pág. 278.

Saussure, F. de (1965): Cours de linguistique générale, Paris, Payot.

Schmidt, S. J. (1990): "Der Radikale Konstruktivismus: Ein neues Paradigma im interdisziplinären Diskurs", en Schmidt, S. J. (ed.): Der Diskurs des Radikalen Konstruktivismus, Frankfurt am Main, Suhrkamp, 1990: 11-88 (trad. esp. En Chico Rico (ed.) (1995), págs. 37-83).

Schröder, H. (1995): "Aspetti semiotici di testi multimediali", en Petöfi y Cicconi (eds.) (1995), págs. 9-24.

Shannon, C. y Weaver, W. (1949): The Mathematical Theory of Communication, Urbana/Champaign (Ill.). University of Illinois Press.

Simone, R. (2001): "Tre paradigmi di scrittura", en Covino, S. (ed.): La scrittura professionale. Ricerca, prassi, insegnamento (= "Atti del I Convegno di studi", Perugina, Università per Stranieri, 23-25 ottobre 2000). Firenze, Olschki, 2001, págs. 33-52.

Talens, J. et al. (1999): Elementos para una semiótica del texto artístico, Madrid, Cátedra.

Teobaldelli, P. (1995): "Per la costruzione di una tipologia dei comunicati multimediali dal punto di vista della testologia semiotica", en Petöfi y Cicconi (eds.) (1995), págs. 35-63.

Timpanaro, S. (1975): Sul materialismo, Pisa, Nistri-Lischi. 
Turchetta, B. (ed.) (1996): Introduzione alla linguistica antropologica, Milano, Mursia.

Van de Velde, R. (1992): Text and Thinking. On Some Roles of Thinking in Text Interpretation, Berlin/New York, W. de Gruyter.

Van Dijk, T. A. (1972a): Some Aspects of Text Grammars, The Hague, Mouton.

Van Dijk, T. A. (1972b): Beiträge zur generativen Poetik, München, Bayerischer Schulbuch-Verlag.

Van Dijk, T. A. (1990): "Discourse \& Society: A new journal for a new research focus", en Discourse \& Society, 1 (1): 5-16.

Van Dijk, T. A. (1994): "Critical Discourse analysis", en Discourse \& Society, 5 (4), págs. 435-436.

Van Dijk, T. A. (1995a): "De la gramática del texto al análisis crítico del discurso", en BELIAR (Boletín de Estudios Lingüísticos Argentinos), 2/6.

Van Dijk, T. A. (1995b): "Discourse semantics and ideology", en Discourse \& Society, 6 (2), págs. 243-289.

Van Dijk, T. A. (ed.) (1997a): Discourse Studies: A Multidisciplinary Introduction. 1: Discourse as Structure and Process, London, Sage.

Van Dijk, T. A. (ed.) (1997b): Discourse Studies: A Multidisciplinary Introduction. 2: Discourse as Social Interaction, London, Sage.

Van Dijk, T. A., Ihwe, R., Petöf, J. S. y Rieser, H. (1972a): "Two Text Grammatical Models. A Contribution to Formal Linguistics and the Theory of Narrative", en Foundations of Language, 8, págs. 499-545.

Van Dijk, T. A., Ihwe, R., Petöfi, J. S. y Rieser, H. (1972b): Zur Bestimmung narrativer Strukturen auf der Grundlage von Textgrammatiken, Hamburg, Buske.

Vitacolonna, L. (1989): Per un paradigma semiotico del testo, Chieti, Vechio Faggio Editore.

Vitacolonna, L. (1991): "Los textos literarios como mundos posibles", en Castilla. Estudios de literatura, 16, págs. 189-212.

Vitacolonna, L. (1999): Principi e contributi di semiotica del testo, Roma, Bulzoni.

Volli, U. (2002): Manuale di semiotica, Roma/Bari, Laterza.

Weinrich, H. (1964): Tempus. Besprochene und erzählte Welt, Stuttgart, Kohlhammer, 1971.

Wodak, R. (1995a): "Critical Linguistics and the Study of Institutional Communication", en Stevenson, P. (ed.): The German Language and the Real World, Oxford, Clarendon Press, 1995: 205-230.

Wodak, R. (1995b): "Critical Linguistics and Critical Discourse Analysis", en Verschueren, J., Östman, J.-O. y Blommaert, J. (eds.): Handbook of Pragmatics, Amsterdam/Philadelphia, Benjamins, 1995, págs. 204-210.

Wodak, R. (1997): "Critical discourse analysis and the study of doctor-patient interacion", en Gunnarsson, B.-L., Linell, P. y Nordberg, B. (eds.): The Construction of Professional Discourse, London/New York, 1997, págs. 173-200. 